\title{
Condensing nuclear pore assembly in oocytes
}

\section{ces \\ Nups establish phase-separated granules}

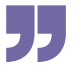

Nuclear pores are multi-protein complexes in the nuclear envelope that form channels enabling selective nucleo-cytoplasmic transport. The building blocks of nuclear pore complexes (NPCs) are nucleoporins (Nups) that include scaffolding Nups, peripheral Nups and pore-lining Nups (known as FG-Nups), which assemble into several sub-complexes. NPC biogenesis at the nuclear envelope had been previously characterized, with NPCs assembling twice during the cell cycle: in the interphase when the nucleus grows, and at the end of mitosis upon nuclear envelope reformation. In addition to the nuclear envelope, NPCs can be present in the endoplasmic reticulum (ER) within structures referred to as annulate lamellae, which are prominent in gametes and embryos. In Drosophila melanogaster embryos, NPCs of annulate lamellae were proposed to serve as a pool of immature NPCs to support rapid embryonic divisions, but how these NPCs assemble away from the nucleus is poorly understood. Hampoelz et al. now propose that the biogenesis of NPCs at annulate lamellae in D. melanogaster oocytes depends on the spatially coordinated formation of phase-separated biomolecular condensates of Nups.

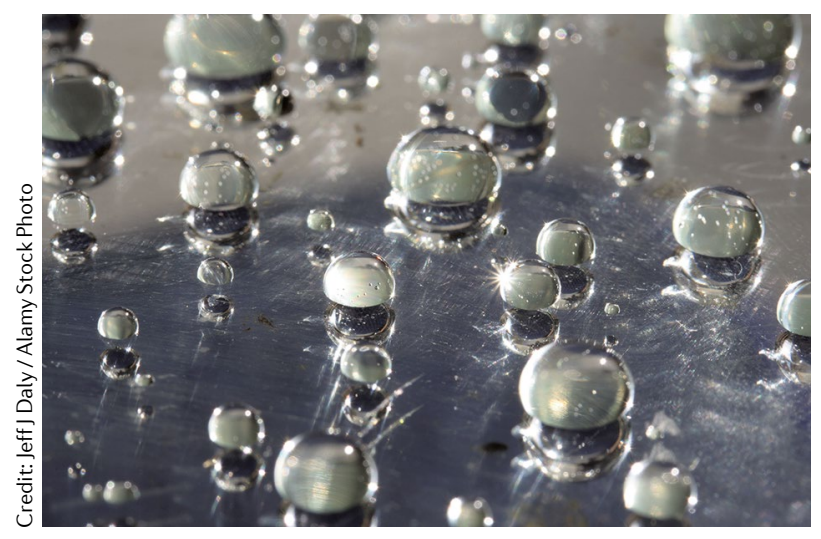

The authors first demonstrated that annulate lamellae are established in the D. melanogaster oocyte and subsequently inherited by the embryo. Imaging of Nups in fly ovaries further revealed that they form granule-like structures. In oocytes, these granules exhibited various sizes that contained scaffolding Nup107 and/or FG-Nups, but were immature, lacking specific NPC components, with the exception of Nup358, which was present in some of the granules. By contrast, nurse cells - oocyte-supporting germline cells - contained mostly large granules predominantly composed of Nup358.

These larger Nup358 granules were motile and were transported to the oocyte from nurse cells. Other Nup granules were also motile, and all these motions depended on intact microtubules. Notably, Nup granules exhibited properties and behaviour of biomolecular condensates, including the ability to fuse and exchange content upon contact. Thus, Nups establish phase-separated granules, the composition of which is differentially regulated between the oocyte and nurse cells. In the oocyte, as these different populations of granules are dynamic, they can intermix to establish NPCs and annulate lamellae.

Ovarian knockdown of Nup358, or of a motor adaptor protein (BicD) that is putatively involved in Nup358 transport, interfered not only with the formation of Nup358 granules in nurse cells, but also with the assembly of annulate lamellae in the oocyte and with embryonic development. Nup358 granules were also shown to serve as platforms for the localized translation of several NPC-associated transcripts, including peripheral Nups (Nup358 and Nup153) and several importins. These data suggest that Nup358 condensates function as a template for the assembly of annulate lamellae, likely by recruiting and stabilizing Nup scaffold components to support NPC biogenesis.

So how do annulate lamellae assemble in the oocyte? The authors hypothesized that this could be driven by the activity of Ran GTPase, which has a well-documented role in NPC assembly at the nuclear envelope. In this context, Ran-GTP releases Nups from their interaction with importin- $\beta$, which otherwise prevents their assembly into NPCs. Ran GTP-activating protein (RanGAP) is also known to bind to Nup358. The model predicts that in the oocyte, Nup358 and the associated RanGAP are progressively diluted from the nurse cell-derived granules. Furthermore, levels of RCC1, the Ran-GTP exchange factor, are high in the ooplasm. These mechanisms would provide means to drive high levels of Ran-GTP specifically in the oocyte, which would generate a pool of Nups available for assembly into NPCs.

In summary, this study provides novel insights into NPC biogenesis in fly ovaries. Here, NPCs form at annulate lamellae, and hence this mechanism of NPC biogenesis is distinct from the two previously described pathways associated with the nuclear envelope. As annulate lamellae have been found in various cell types, they might contribute to NPC homeostasis more broadly.

Paulina Strzyz

ORIGINAL ARTICLE Hampoelz, B. et al. Nuclear pores assemble from nucleoporin condensates during oogenesis. Cell 179, 671-686 (2019) RELATED ARTICLES Beck, M. \& Hurt, E.

The nuclear pore complex: understanding its function through structural insight. Nat. Rev. Mol. Cell Biol. 18, 73-89 (2017) |Ungricht, R. \& Kutay, U. Mechanisms and functions of nuclear envelope remodelling. Nat. Rev. Mol. Cell Biol. 18, 229-245 (2017) | Banani, S. et al. Biomolecular condensates: organizers of cellular biochemistry. Nat. Rev. Mol. Cell Biol. 18, 285-298 (2017) 